MMCA-97 Conference, pp. 84-91

R. Ciegis (Ed)

(C) 1997 Vilniaus Gedimino technikos universitetas

\title{
EFFECTIVE FINITE-DIFFERENCE METHODS FOR THE SOLUTIONS OF FILTRATION PROBLEMS IN MULTILAYER DOMAINS
}

\section{H. KALIS}

University of Latvia, Departament of Physics and Mathematics, 19 Blv. Rainis, Riga, LV-1586, Latvia,

e-mail:kalis@fmf.lu.lv

\begin{abstract}
In papers $[1,2]$ there were consider different assumptions for averaging methods along the vertical coordinate.These methods were applied for the mathematical simulation of the mass transfer process in multilayered underground systems. A specific feature of these problems is that it is necessity to solve the 3-D initial-boundary-value problems for parabolic type partial differential equations of second order with piece-wise parameters in multilayer domain. Therefore here an effective finite-difference method for solving a problem of the above type is developed.This method may be considered as a generalization of the method of finite volumes [3] for the layered systems. In the case of constant piece-wise coefficients we obtain the exact discrete approximation of steady-state 1-D boundary-value problem. This procedure allows to reduce the 3 -D problem to a system of 2-D problems and the 2-D problem to a system of 1-D problems.
\end{abstract}

\section{FORMULATION OF PROBLEM}

The process of filtration we will consider in 3 -D domain of parallelepiped $D=\left\{(x, y, z):(x, y) \in \Omega, H_{0} \leq z \leq H_{N}\right\}$, where $\Omega=\left\{(x, y):-l_{x} \leq x \leq\right.$ $\left.l_{x},-l_{y} \leq y \leq l_{y}\right\}$ is rectangle in the horizontal $x, y$ directions with length of edges $2 l_{x}, 2 l_{y}, H_{N}-H_{0}$ is the height of domain in the vertical $z$ - direction. The domain $D$ consist of multilauer medium of $\mathrm{N}$ layers (cylinders)

$$
D_{k}=\left\{(x, y, z):(x, y) \in \Omega, H_{k-1}<z<H_{k}\right\} \quad k=\overline{1, N},
$$

with horizontal interfaces

$$
S_{k}=\left\{\left(x, y, H_{k}\right):(x, y) \in \Omega\right\} \quad k=\overline{1, N-1},
$$


where $H_{k}-H_{k-1}$ is the height of layer $D_{k}$. We will to find the distribution of physical magnitudes $u_{k}=u_{k}(x, y, z, t)$ in every layer $D_{k}$ at the point $(x, y, z) \in$ $D_{k}$ and time $t>0$ by solving the partial differential equation in the following form [2]

$$
\partial\left(\lambda_{k} \partial u_{k} / \partial z\right) / \partial z+L_{k}\left(u_{k}\right)=-F_{k}(x, y, z, t) \quad k=\overline{1, N},
$$

where $F_{k}$ is continuously differentiable function of external sources in every layer $D_{k}$.The physical parameters $\lambda_{k}$ of heat conductivity, diffusion coefficients or coefficients of filtration and the differential operator $L_{k}$ are depends only of $\mathrm{x}, \mathrm{y}, \mathrm{t}\left(\lambda_{k}>0\right)$. We assume that the function $\lambda_{k}$ and the coefficients of differential operator are piece-wise constants functions of the vertical coordinate $z$ with discontinuity on the surface $S_{k}$. The operator $L_{k}$ we can consider in the form

$$
\begin{aligned}
& L_{k}\left(u_{k}\right)=\partial\left(\lambda_{k} \partial u_{k} / \partial x\right) / \partial x+\partial\left(\lambda_{k} \partial u_{k} / \partial y\right) / \partial y \\
& \quad-d_{k} \partial u_{k} / \partial t-a_{k} \partial u_{k} / \partial x-b_{k} \partial u_{k} / \partial y-c_{k} u_{k} \quad k=\overline{1, N}
\end{aligned}
$$

where an example $w_{k}=\left(a_{k}, b_{k}\right)$ is the velocity of filtering for the flow of the underground water in layer $D_{k}, d_{k}, c_{k}$ mass transfer coefficients $\left(d_{k}>0\right)$. The equations $(3,4)$ are considered in every layer $D_{k}$ of different properties of medium. The physical magnitude $u_{k}$ and the flux $\lambda_{k} \partial u_{k} / \partial z$ must be continuous on the interior boundary. Therefore we have on the $S_{k}$ following continuity conditions

$$
u_{k}=u_{k+1}, \quad \lambda_{k} \partial u_{k} / \partial z=\lambda_{k+1} \partial u_{k+1} / \partial z, \quad k=\overline{1, N-1} .
$$

We assume that the whole N-layered system is bounded above and below with the plane surfaces $S_{0}, S_{N}(2)$. The boundary conditions on the $S_{0}, S_{N}$ may be written corresponding as

$$
\begin{gathered}
\nu_{0} \lambda_{1} \partial u_{1} / \partial z-\alpha_{0} u_{1}=-\alpha_{0} \Phi_{0}(x, y, t), \\
\nu_{1} \lambda_{N} \partial u_{N} / \partial z+\alpha_{N} u_{N}=\alpha_{N} \Phi_{1}(x, y, t),
\end{gathered}
$$

where $(x, y) \in \Omega, t \geq 0$,

$\nu_{0}=0$ or $\nu_{1}=0$ for the corresponding Dirichlet boundary conditons: $u_{1}=\Phi_{0}$ or $u_{N}=\Phi_{1}$

$\nu_{0}=1$ or $\nu_{1}=1$ for the corresponding Neumann $\left(\alpha_{0}=0\right.$ or $\left.\alpha_{N}=0\right)$ or general form of boundary conditions;

$\alpha_{0} \geq 0, \alpha_{N} \geq 0, \Phi_{0}, \Phi_{1}$ are given continuosly differentiable functions. The equations $(3,4)$ with conditions (5-7) along the z-coordinate has been solved in a domain $D$ with different boundary conditions in the $x, y$ directions at $x= \pm l_{x} ; y= \pm l_{y}$ and with initial condition at $t=0$ in the case of time depending problem.The form of this conditions are not assential for obtaining 
the numerical algorithm.

\section{FINITE VOLUMES METHOD}

The approximation of differential problem is based on the conservation law approch.Therefore it develops the monotonous difference scheme using a physical conservation law. This method is based of the application of the method of finite volumes [3]. We consider the nonuniform grid in the z-direction placed on the interval $\left(H_{0}, H_{N}\right)$ with blocks centered at the grid points $z_{j}, j=$ $\overline{1, M}, \quad M \geq N \quad\left(z_{0}=H_{0}, z_{M}=H_{N}\right)$. We shall refer to the endpoints of the interval about the point $z_{j}$ as $z_{j \pm 0.5}$. This interval $\left(z_{j-0.5}, z_{j+0.5}\right)$ is refered to as the control volume associated with the grid point $z_{j}$ (the jth cell). The grid contain the z-coordinates $H_{k}$ of surfaces $S_{k}, k=\overline{0, N}$ and in addition some grid points in layers $D_{k}, k=\overline{1, N}$ when this is necessary for demonstrating the behaviour of discrete solution in this layers. To derive a difference equation associated with the $\mathrm{j}$-th grid point $z_{j}$ we integrate differential equation (3) to the $\mathrm{j}$-th cell.We define the functions $G_{k}=-\left(F_{k}+L_{k}\left(u_{k}\right)\right), \quad W=\lambda \partial u / \partial z, \quad W_{j \pm 0.5}$ and the integrals $B_{j}=\left(\lambda_{j}\right)^{-1} \int_{z_{j-1}}^{z_{j}} d z \int_{z_{j-0.5}}^{z} G_{j} d \xi$, where $W_{j \pm 0.5}=\left.W\right|_{z=z_{j \pm 0.5}}, \quad h_{j}=z_{j}-z_{j-1}, \quad z_{j \pm 0.5}=\left(z_{j}+z_{j \pm 1}\right) / 2$.

We shall consider corresponding from central grid point $z_{j} 4$ cases for the applying the finite volumes method: $z_{j} \in S_{k}, k=\overline{1, N-1} ; \quad z_{j} \in D_{k}, k=\overline{1, N}$ ; $z_{j} \in S_{0}$ if $\nu_{0}=1$ and $z_{j} \in S_{N}$ if $\nu_{1}=1$.

2.1. Let $z_{j}=H_{k} \in S_{k}$ is the central grid point and $z_{j-1}, z_{j+1}$ are the others grid points. We integrate the equation (3) from $z_{j-0.5}$ to $z_{j}$ and $z_{j}$ to $z_{j+0.5}$. We get

$$
W_{j+0.5}-W_{j-0.5}=\int_{z_{j-0.5}}^{z_{j}} G_{j} d z+\int_{z_{j}}^{z_{j+0.5}} G_{j+1} d z
$$

where $\left.W\right|_{z_{j}-0}=\left.W\right|_{z_{j}+0}$.

This is the integral form of the conservation law to the interval $\left(z_{j-0.5}, z_{j+0.5}\right)$. In the classical formulation for finite volumes method [3] it is assumed that the flux terms $W_{j \pm 0.5}$ in (8) are approximated with the difference expressions. Then the corresponding difference scheme is not exact for given functions $G_{j}$. Here we have the possibility to make the exact difference scheme. Therefore we integrate equation (3) from $z_{j-0.5}$ to $z \in\left(z_{j-1}, z_{j}\right)$, divid this expression by $\lambda_{k}$ and integrating from $z_{j-1}$ to $z_{j}$. We obtain $u_{k}\left(z_{j}\right)-u_{k}\left(z_{j-1}\right)=$ $\left(A_{j}\right)^{-1} W_{j-0.5}+B_{j}$, where $A_{j}=\lambda_{k} / h_{j}$ and $u_{k}\left(z_{j}\right), u_{k}\left(z_{j-1}\right)$ represents the value of function $u_{k}$ at $z_{j}, z_{j-1}$. Hence

$$
W_{j-0.5}=A_{j}\left(u_{k}\left(z_{j}\right)-u_{k}\left(z_{j-1}\right)\right)-A_{j} B_{j} .
$$


Similarly the flux term

$$
W_{j+0.5}=A_{j+1}\left(u_{k+1}\left(z_{j+1}\right)-u_{k+1}\left(z_{j}\right)\right)-A_{j+1} B_{j+1},
$$

where $A_{j+1}=\lambda_{k+1} / h_{j+1}$ and $u_{k+1}\left(z_{j}\right), u_{k+1}\left(z_{j+1}\right)$ are the value of function $u_{k+1}$ at $z_{j}, z_{j+1}$. To derive a 3 -point difference equation associated with the central grid point $z_{j}=H_{k}$ we want to apply equation (8) in the form

$$
A_{j+1}\left(u_{k+1}\left(z_{j+1}\right)-u_{k+1}\left(z_{j}\right)\right)-A_{j}\left(u_{k}\left(z_{j}\right)-u_{k}\left(z_{j-1}\right)\right)=R_{j}
$$

where $R_{j}=R_{j}^{-}+R_{j}^{+}, R_{j}^{-}=\left(h_{j}\right)^{-1} \int_{z_{j-1}}^{z_{j}}\left(z-z_{j-1}\right) G_{j} d z$,

$R_{j}^{+}=\left(h_{j+1}\right)^{-1} \int_{z_{j}}^{z_{j+1}}\left(z_{j+1}-z\right) G_{j+1} d z$.

2.2.If $z_{j} \in D_{k}, h_{j}=h_{j+1}$, then difference equation (11) associated with point $z_{j}$ has the form

$$
\lambda \delta_{z}^{2}\left(u_{k}\right)_{j}=h_{j}^{-1} R_{j},
$$

where $\delta_{z}^{2}(v)_{j}=\left(v_{j+1}-2 v_{j}+v_{j-1}\right) / h_{j}^{2}$ denotes a central difference expression of second order for approximation the derivative $\partial^{2} v / \partial z^{2}$ at the central grid point $z_{j}$.

2.3. Let $z_{j}=z_{0}=H_{0} \in S_{0}$ and $\nu_{0}=1$. We apply the integral form of the conservation law to the half interval $\left(z_{0}, z_{0.5}\right)$ marked off to the right of the boundary point $z_{0}$. We get

$$
A_{1}\left(u_{1}\left(z_{1}\right)-u_{1}\left(z_{0}\right)\right)-\alpha_{0}\left(u_{1}\left(z_{0}\right)-\Phi_{0}\right)=R_{0},
$$

where $A_{1}=\lambda_{1} / h_{1}$ and $u_{1}\left(z_{0}\right), u_{1}\left(z_{1}\right)$ represents the value of function $u_{1}$ at $z_{0}, z_{1}, R_{0}=\left(h_{1}\right)^{-1} \int_{z_{0}}^{z_{1}}\left(z_{1}-z\right) G_{1} d z$.

2.4. If $z_{j}=H_{N} \in S_{N}, \nu_{1}=1$, then similarly in advance we obtain

$$
-\alpha_{N}\left(u_{N}\left(H_{N}\right)-\Phi_{1}\right)-A_{N}\left(u_{N}\left(H_{N}\right)-u_{N}\left(z_{j-1}\right)\right)=R_{N},
$$

where $A_{N}=\lambda_{N} / h_{N}$ and $u_{N}\left(z_{j-1}\right)$ represent the value of function $u_{N}$ at $z_{j-1}$, $R_{N}=\left(h_{N}\right)^{-1} \int_{z_{j-1}}^{H_{N}}\left(z-z_{j-1}\right) G_{N} d z$.

We see that the difference equations (11-14) are exact approximations for solving steady-state 1-D boundary - value problem (3),(5 - 7) depending only of $z,\left(L_{k}\left(u_{k}\right)=0, l_{x}=l_{y}=\infty, c_{k}=0\right)$.

\section{ONE-DIMENSIONAL EXACT DIFFERENCE SCHEME}

Suppose that $L_{k}\left(u_{k}\right)=0, u_{k}=u_{k}(z), F_{k}=F_{k}(z), \quad \lambda_{k} ; \Phi_{0} ; \Phi_{1}$ are constants and the grid points are $z_{k}=H_{k}, k=\overline{0, N}$. If $v_{j}=u_{j}\left(z_{j}\right)$ is the value of function $u_{j}$ at the grid point $z_{j}, j=\overline{0, N}$, then,evaluating the integral $R_{j}$ in 
the right side of (11-14), one obtains exact 1-D steady-state difference scheme $\left(\nu_{0}=\nu_{1}=1\right)$

$$
A_{j+1}\left(v_{j+1}-v_{j}\right)-A_{j}\left(v_{j}-v_{j-1}\right)=R_{j}, \quad j=\overline{0, N},
$$

where $A_{0}=\alpha_{0} \geq 0, A_{N+1}=\alpha_{N} \geq 0, v_{-1}=\Phi_{0}, v_{N+1}=\Phi_{1}, A_{j}=\lambda_{j} / h_{j}>$ $0, j=\overline{1, N}$. Therefore, the difference scheme (15) is monotone and has an unique solution [4]. We can to consider in addition the new grid points or interpolating points for approximationof functions $u_{k}$ in layers $D_{k}$.In the case of uniform grid we use the difference equations (12). The finite-difference scheme (15) can be solving by factorisation method for tri-diagonal matrix (Thomas algorithm [3]).

\section{SOLUTION OF ONE-DIMENSIONAL PROBLEM}

We can solve the difference scheme (15) also in a more simplest form. For this purpose from the first equation (15) we conclude that $A_{1}\left(v_{1}-v_{0}\right)-\alpha_{1}^{+}\left(v_{1}-\right.$ $\left.\Phi_{0}\right)=\alpha_{1}^{+}\left(\alpha_{0}\right)^{-1} R_{0}$, where $\left.\alpha_{1}^{+}\right)^{-1}=\left(\alpha_{0}\right)^{-1}+A_{1}^{-1}$ is the inverse value of the interaction coefficient of the two layers in directe direction. Further more, from the second equations (15) follows $A_{2}\left(v_{2}-v_{1}\right)-A_{1}\left(v_{1}-v_{0}\right)=R_{1}$. Therefore $A_{2}\left(v_{2}-v_{1}\right)-\alpha_{1}^{+}\left(v_{1}-\Phi_{0}\right)=\alpha_{1}^{+} R_{1}^{+}$, where $R_{1}^{+}=R_{1} / \alpha_{1}^{+}+R_{0} / \alpha_{0}$. Hence

$$
A_{m+1}\left(v_{m+1}-v_{m}\right)-\alpha_{m}^{+}\left(v_{m}-\Phi_{0}\right)=\alpha_{m}^{+} R_{m}^{+},
$$

where

$$
\begin{aligned}
& \left(\alpha_{m}^{+}\right)^{-1}=\left(\alpha_{m-1}^{+}\right)^{-1}+A_{m}^{-1}=\left(\alpha_{0}\right)^{-1}+A_{1}^{-1}+\cdots+A_{m}^{-1} \\
& R_{m}^{+}=R_{m} / \alpha_{m}^{+}+R_{m-1}^{+} / \alpha_{m-1}^{+}=R_{0} / \alpha_{0}+R_{1} / \alpha_{1}^{+}+\cdots+R_{m} / \alpha_{m}^{+} \\
& m=\overline{1, N-1}
\end{aligned}
$$

From the last equation (15) and from (16) for $m=N-1$ follows $\alpha_{N-1}^{-}\left(\Phi_{1}-\right.$ $\left.v_{N-1}\right)-\alpha_{N-1}^{+}\left(v_{N-1}-\Phi_{0}\right)=R_{N-1}^{ \pm}$and

$$
v_{N-1}=\frac{\alpha_{N-1}^{-} \Phi_{1}+\alpha_{N-1}^{+} \Phi_{0}-R_{N-1}^{ \pm}}{\alpha_{N-1}^{-}+\alpha_{N-1}^{+}},
$$

where $R_{N-1}^{ \pm}=R_{N-1}^{+} \alpha_{N-1}^{+}+\alpha_{N-1}^{-}\left(\alpha_{N}\right)^{-1} R_{N}$. Similarly can be obtained $v_{N}=$ $\left(\alpha_{N} \Phi_{1}+\alpha_{N}^{+} \Phi_{0}-R_{N}^{ \pm}\right) /\left(\alpha_{N}+\alpha_{N}^{+}\right)$, where $R_{N}^{ \pm}=R_{N}+\alpha_{N}^{+} R_{N-1}^{+}$. For Dirichlet boundary condition $\left(\nu_{0}=0\right.$ or $\left.\nu_{1}=0\right)$ we can take $\alpha_{0}=\infty$ or $\alpha_{N}=\infty$ $\left(v_{0}=\Phi_{0}\right.$ or $\left.v_{N}=\Phi_{1}\right)$.

We can also consider the opposite direction. Then similary (16) follows

$$
\alpha_{N-n}^{-}\left(\Phi_{1}-v_{N-n}\right)-A_{N-n}\left(v_{N-n}-v_{N-n-1}\right)=R_{N-n}^{-} \alpha_{N-n}^{-}
$$


where $\left(\alpha_{N-n}^{-}\right)^{-1}=\left(\alpha_{N}\right)^{-1}+\left(A_{N}\right)^{-1}+\cdots+\left(A_{N-n+1}\right)^{-1}$,

$R_{N-n}^{-}=R_{N-n} / \alpha_{N-n}^{-}+R_{N-n+1} / \alpha_{N-n+1}^{-}+\cdots+R_{N} / \alpha_{N}$.

From the first equation (15) and from (18) by $n=N-1$ follows

$\alpha_{1}^{+}\left(\Phi_{0}-v_{1}\right)-A_{1}\left(v_{1}-v_{0}\right)=\alpha_{1}^{+}\left(\alpha_{0}\right)^{-1} R_{0}$ and

$$
v_{1}=\frac{\alpha_{1}^{-} \Phi_{1}+\alpha_{1}^{+} \Phi_{0}-R_{1}^{ \pm}}{\alpha_{1}^{-}+\alpha_{1}^{+}}
$$

where $R_{1}^{ \pm}=R_{1}^{-} \alpha_{1}^{-}+\alpha_{1}^{+}\left(\alpha_{0}\right)^{-1} R_{0}$. The value of $v_{0}$ can be obtained in the form $v_{0}=\left(\alpha_{0}^{-} \Phi_{1}+\alpha_{0} \Phi_{0}-R_{0}^{ \pm}\right) /\left(\alpha_{0}^{-}+\alpha_{0}\right)$, where $R_{0}^{ \pm}=R_{0}+R_{1}^{-} \alpha_{0}^{-} \cdot$ ¿From the expression (16),(18) by $m=k-1$ and $n=N-k$ follows $\alpha_{k}^{+}\left(\Phi_{0}-v_{k}\right)+$ $A_{k}\left(v_{k}-v_{k-1}\right)=\alpha_{k}^{+} R_{k-1}^{+}$and

$$
v_{k}=\frac{\alpha_{k}^{-} \Phi_{1}+\alpha_{k}^{+} \Phi_{0}-R_{k}^{ \pm}}{\alpha_{k}^{-}+\alpha_{k}^{+}}
$$

where $R_{k}^{ \pm}=R_{k}^{-} \alpha_{k}^{-}+\alpha_{k}^{+} R_{k-1}^{+}, \quad k=\overline{1, N-1}$.

We can consider in addition for example the grid point $z_{i}$ with steps $h_{i}^{+}, h_{i}^{-}$ between the nearests grid points $z_{i} \pm h_{i}^{ \pm}$. Then we have also the terms in the corresponding summ

$$
\begin{aligned}
& \left(\alpha_{k}^{+}\right)^{-1}=\left(\alpha_{0}\right)^{-1}+A_{1}^{-1}+\cdots+h_{i}^{-} / \lambda_{i}^{-}+h_{i}^{+} / \lambda_{i}^{+} \cdots+A_{k}^{-1}, \\
& \left(\alpha_{k}^{-}\right)^{-1}=\left(\alpha_{N}\right)^{-1}+A_{N}^{-1}+\cdots+h_{i}^{-} / \lambda_{i}^{-}+h_{i}^{+} / \lambda_{i}^{+} \cdots+A_{k+1}^{-1}, \\
& R_{k}^{+}=R_{0} / \alpha_{0}+R_{1} / \alpha_{1}^{+}+\cdots+R_{i} / \alpha_{i}^{+}+\cdots+R_{k} / \alpha_{k}^{+}, \\
& R_{k}^{-}=R_{N} / \alpha_{N}+\cdots+R_{i} / \alpha_{i}^{-}+\cdots+R_{k} / \alpha_{k}^{-}, \\
& \text {where } \lambda_{i}^{-}, \lambda_{i}^{+} \text {are the corresponding parameters of layers. }
\end{aligned}
$$

\section{DISCRETE APPROXIMATION OF FIRST AND SECOND ORDER}

If $L_{k}\left(u_{k}\right) \neq 0$ and the functions $\lambda, F_{k}, \Phi_{0}, \Phi_{1}$ are depends of others variable, then the difference scheme (15) is not exact ( this is the case of 2-D or 3-D problems with $\left.l_{x} \neq \infty, l_{y} \neq \infty\right)$. In this cases is the accuracy of order $O\left(h_{x}+h_{y}+h_{z}\right)$ or $O\left(h_{x}^{2}+h_{y}^{2}+h_{z}^{2}\right)$, where $h_{x}, h_{y}, h_{z}$ are the steps of uniform grid in the corresponding directions. We consider different approximations for right side function $R_{j}$ in equations (11-14).

5.1. To approximate $R_{j}$ of (11) on the nonuniform we consider the following Taylor series expansions of function $G_{k}$ :

$G_{k}(z)=G_{k}\left(z_{j}\right)+\left(z-z_{j}\right) G_{k}^{\prime}\left(z_{j}\right)+O\left(z-z_{j}\right)^{2}, z \in\left(z_{j-1}, z_{j}\right)$

$G_{k+1}(z)=G_{k+1}\left(z_{j}\right)+\left(z-z_{j}\right) G_{k+1}^{\prime}\left(z_{j}\right)+O\left(z-z_{j}\right)^{2}, z \in\left(z_{j}, z_{j+1}\right)$

where $G_{k}^{\prime}=\partial G_{k} / \partial z, z_{j}=H_{k}$.

Then $R_{j}=\frac{1}{2}\left(G_{k+1}\left(z_{j}\right) h_{j+1}+G_{k}\left(z_{j}\right) h_{j}\right)+\frac{1}{6}\left(G_{k+1}^{\prime}\left(z_{j}\right) h_{j+1}^{2}-G_{k}^{\prime}\left(z_{j}\right) h_{j}^{2}\right)+O\left(h^{3}\right)$, where $h=\max \left(h_{j}, h_{j+1}\right), 0<z_{j}<H_{N}$. Since $G_{k+1}^{\prime}=\left(G_{k+1}\left(z_{j+1}\right)-\right.$ $\left.G_{k+1}\left(z_{j}\right)\right) / h_{j+1}+O\left(h_{j+1}\right)$, 
$G_{k}^{\prime}=\left(G_{k}\left(z_{j}\right)-G_{k}\left(z_{j-1}\right)\right) / h_{j}+O\left(h_{j}\right)$ the expression can to obtain in the form

$$
R_{j}=\frac{h_{j+1}}{6}\left(G_{k+1}\left(z_{j+1}\right)+2 G_{k+1}\left(z_{j}\right)\right)+\frac{h_{j}}{6}\left(G_{k}\left(z_{j-1}\right)+2 G_{k}\left(z_{j}\right)\right)+O\left(h^{3}\right) .
$$

With $h_{j}=h_{j+1}$ in the case of uniform grid the expression for $R_{j}$ can be rewritten in the form

$$
R_{j}=((G))_{j} h_{j}+\frac{1}{6}\left[G^{\prime}\right]_{j} h_{j}^{2}+O\left(h_{j}^{3}\right),
$$

where $((G))_{j}=\left(G_{k+1}\left(z_{j}\right)+G_{k}\left(z_{j}\right)\right) / 2$ is the averaged value of $G$; $\left.\left[G^{\prime}\right]_{j}=G_{k+1}^{\prime}\left(z_{j}\right)-G_{k}^{\prime}\left(z_{j}\right)\right)$ is the jump of $G^{\prime}$ at the point $z_{j}$. In this case $\left(z_{j}=H_{k}\right)$ :

$$
\begin{gathered}
((G))_{j}=-\left[((F))_{j} h_{j}+\partial\left(((\lambda)) \partial u_{k} / \partial x\right) / \partial x+\partial\left(((\lambda)) \partial u_{k} / \partial y\right) / \partial y\right. \\
\left.-((d)) \partial u_{k} / \partial t-((a)) \partial u_{k} / \partial x-((b)) \partial u_{k} / \partial y-((c)) u_{k}\right]
\end{gathered}
$$

where

$((F))_{j}=\left(h_{j}\right)^{-1} \int_{z_{j-1}}^{z_{j}}\left(z-z_{j-1}\right) F_{j} d z+\left(h_{j+1}\right)^{-1} \int_{z_{j}}^{z_{j+1}}\left(z_{j+1}-z\right) F_{j+1} d z$.

The expressions $(21,22)$ approximates $R_{j}$ to the second order in $h_{j}$.

5.2. From (13) evaluating $R_{0}$ we se, using a Taylor series expansion, that $G_{1}(z)=G_{1}\left(z_{0}\right)+\left(z-z_{0}\right) G_{1}^{\prime}\left(z_{0}\right)+O\left(z-z_{0}\right)^{2}, z \in\left(z_{0}, z_{1}\right)$.

So $R_{0}=0.5\left(G_{1}\left(z_{0}\right) h_{1}+\left(G_{1}^{\prime}\left(z_{0}\right) h_{1}^{2} / 6+O\left(h_{1}^{3}\right)\right.\right.$ or

$$
R_{0}=\frac{h_{1}}{6}\left(G_{1}\left(z_{1}\right)+2 G_{1}\left(z_{0}\right)\right)+O\left(h_{1}^{3}\right) .
$$

The expressions (24) approximate $R_{0}$ to the second order in $h_{1}$.

5.3. Similarly from (14), evaluating $R_{N}$ we can show that

$R_{N}=0.5\left(G_{N}\left(H_{N}\right) h_{N}-\left(G_{N}^{\prime}\left(H_{N}\right) h_{N}^{2} / 6+O\left(h_{N}^{3}\right)\right.\right.$, or

$$
R_{N}=\frac{h_{N}}{6}\left(G_{N}\left(z_{j-1}\right)+2 G_{N}\left(H_{N}\right)\right)+O\left(h_{N}^{3}\right) .
$$

The second order of accuracy in $x, y$ directions can be obtain by the central difference approximation for derivatives in the expressions $(21-25)$. If $a_{k} \neq 0, b_{k} \neq 0$ then the monotonous difference schemes can be consider [4].

\section{SOME EXAMPLES}

In the following examples we discuss the applications the finite- difference scheme (15). 
6.1. We assume that the boundary-value problem of mathematical physics (3) - (7) for the two-layered system $(N=2)$ is steady-state $\left(c_{k}=0\right)$ with the boundary conditions at the side $x= \pm l_{k}, y= \pm l_{y}$ :

$\partial u_{k} / \partial x=\partial u_{k} / \partial y=0$. Let $H_{0}=0, H_{1}=\epsilon, H_{2}=1, \nu_{0}=1, \nu_{1}=0$, $\alpha_{0}=\alpha_{2}=1, F_{1}=-\epsilon^{-1}, F_{2}=0, \Phi_{0}=\Phi_{1}=0$. Then from (15) follows the system of two equations

$\lambda_{1} \epsilon^{-1}\left(v_{1}-v_{0}\right)-\alpha_{0} v_{0}=R_{0}, \quad-\lambda_{2}(1-\epsilon)^{-1} v_{1}-\lambda_{1} \epsilon^{-1}\left(v_{1}-v_{0}\right)=R_{1}$, where $R_{0}=\epsilon^{-1} \int_{0}^{\epsilon}(1-z / \epsilon) d z=\epsilon^{-1} \int_{0}^{\epsilon} z / \epsilon d z=0.5$.

We obtain the exact values of solution at the 3 grid points $z_{0}=0, z_{1}=\epsilon, z_{2}=$ 1 in the form

$v_{0}=\left((1-\epsilon) \lambda_{1}+0.5 \epsilon \lambda_{2}\right) / p, \quad v_{1}=0.5(\epsilon-1)\left(\alpha_{0} \epsilon+2 \lambda_{1}\right) / p, v_{2}=0$,

where $p=\alpha_{0} \lambda_{1}(1-\epsilon)+\lambda_{2}\left(\lambda_{1}+\alpha_{0} \epsilon\right)$.

6.2 We consider the 2-dimensional steady -state process with conditions

$\partial u_{k} /\left.\partial x\right|_{x= \pm l_{x}}=0, u_{k}=u_{k}(y, z), b_{k}=c_{k}=0, F_{k}=F_{k}(y, z), \lambda_{k}=\mathrm{const}$ and of uniform grid in the $y$ direction with points $y_{i}=-l_{y}+i h_{y}, i=$ $\overline{0,2 N_{y}}\left(h_{y} N_{y}=l_{y}\right)$. Since the functions $\lambda_{k}, F_{k}, \Phi_{0}, \Phi_{1}$ are continously differentiable in the $x, y, t$ directions the continuity condition (5) can to derivate with respect to $x, y, t$ one or more time.Then from (15), (23-25) follows the finite-difference scheme

$\nu_{0} \lambda_{1} h_{1}^{-2}\left(v_{i, 1}-v_{i, 0}\right)-\alpha_{0} h_{1}^{-1}\left(v_{i, 0}-\left(\Phi_{0}\right)_{i}\right)+\nu_{0}\left(\lambda_{1} / 2\right.$

$\left.+h_{1} \alpha_{0} / 6\right) \delta_{y}^{2}\left(v_{0}\right)_{i}=\left(F_{0}^{*}\right)_{i} \quad i=\overline{1,2 N_{y}-1}$;

$\Lambda_{z} v_{i, j}+\Lambda_{y} v_{i, j}=-\left(F_{j}^{*}\right)_{i}, \quad j=\overline{1, N-1}, i=\overline{1,2 N_{y}-1}$

$\alpha_{N} h_{j}^{-1}\left(\left(\Phi_{1}\right)_{i}-v_{i, N}\right)-\nu_{1} \lambda_{N} h_{N}^{-2}\left(v_{i, N}-v_{i, N-1}\right)$

$+\nu_{1}\left(\lambda_{N} / 2+h_{N} \alpha_{1} / 6\right) \delta_{y}^{2}\left(v_{N}\right)_{i}=\left(F_{N}^{*}\right)_{i}, \quad i=\overline{1,2 N_{y}-1}$,

where

$v_{i, j}=u_{j}\left(y_{i}, z_{j}\right),(p)_{i}=\left.p\right|_{y=y_{i}}, \delta_{y}^{2}(p)_{i}=\left((p)_{i+1}-2(p)_{i}+(p)_{i-1}\right) / h_{y}^{2}, p=$ $F_{j} ; v_{j} ; \Phi_{0} ; \Phi_{1},\left(F_{0}^{*}\right)_{i}=\nu_{0}\left(\alpha_{0} h_{1} \delta_{y}^{2}\left(\Phi_{0}\right)_{i} / 6-\left(F_{1}\right)_{i} / 2-h_{1}\left(F_{1}^{\prime}\right)_{i} / 6\right)$,

$\left(F_{N}^{*}\right)_{i}=\nu_{1}\left(\alpha_{N} h_{N} \delta_{y}^{2}\left(\Phi_{1}\right)_{i} / 6-\left(F_{N}\right)_{i} / 2+h_{N}\left(F_{N}^{\prime}\right)_{i} / 6\right)$,

$\left(F_{j}^{*}\right)_{i}=\left(\left(F_{j}\right)_{i} h_{j}+\left(F_{j+1}\right)_{i} h_{j+1}\right) /\left(2 \hbar_{j}\right)$,

$\Lambda_{z} v_{i, j}=\left(\hbar_{j}\right)^{-1}\left(\lambda_{j+1} / h_{j+1}\left(v_{i, j+1}-v_{i, j}\right)-\lambda_{j} / h_{j}\left(v_{i, j}-v_{i, j-1}\right)\right)$,

$\Lambda_{y} v_{i, j}=\left(\lambda_{j} h_{j}+\lambda_{j+1} h_{j+1}\right) /\left(2 \hbar_{j}\right) \delta_{y}^{2}\left(v_{j}\right)_{i}$,

$\hbar_{j}=0.5\left(h_{j}+h_{j+1}\right), \quad F_{j}^{\prime}=\partial F_{j} /\left.\partial z\right|_{z=z_{j}}$.

\section{REFERENCES}

[1] J. Bear, Hydraulic of groundwater, Mc.Graw-Hill Inc., 1979, 569 p.

[2] A. Buikis, The analysis of schemes for the modelling same processes of filtration in the underground, Riga, Acta Universitatis Latviensis,vol.592, 1994, 25-32(in Latvian).

[3] J.W. Thomas, Numerical partial differential equations, Finite difference methods, Springer- Verlag, New-York,Inc.,1995,437 p.

[4] H. Kalis, Ausarbeitung und Anwendung der spezialen numerischen Methoden zur Losung der Probleme der mathemtischen Physik,Hydrodinamik und Magnetohydrodynamik, Riga, Acta Universitatis Latviensis, vol.588, 1993, P. 175-206. 